\title{
Introduction of Medical Device Development through Industry-Academia Collaboration by the Hamamatsu Method
}

\author{
Yuko AmAno-Ito*,\#
}

\begin{abstract}
To develop medical devices, collaborations are necessary between universities and hospitals that have technological problems and companies with the appropriate technological facilities to solve these problems. Large companies do not generally compete in niche markets, where large sales and profits are not expected, which then become the target for small and medium enterprises (SMEs). In this study, the "Mono-Dzukuri region" was considered because of its dense concentration of universities or hospitals and SMEs. However, interventions by industry-academia collaboration coordinators (CDs) are required to ensure smooth collaborations between academia and SMEs that are unfamiliar with medical device development as well as companies that lack market presence in the medical device field. In this study, the Hamamatsu area, a locality that is actively engaged in medical device development through collaborations, was evaluated for different hypotheses. The CDs are integral to forming an appropriate ecosystem for the development team to educate doctors, medical workers, SMEs, and venture companies. Specifically, seminars on medical and clinical research methods are used to educate stakeholders on the importance of collaboration. These seminars provide collaborative opportunities, $R \& D$ expenses, and technology transfer and sales promotion support from commercialization to sales. Local governments and banks also support these efforts. CDs from different institutions also actively collaborate with each other; they use co-working spaces and the Coordinator Support System to share information and consult on problems. Through these efforts, CDs have successfully assisted the continuous development of medical devices. Both successful and failed commercialization cases are reported herein. After examining the causes of failure, it was found that collaborations often failed when the needs of the marketing authorization holders could not be met and when the medical and technical needs were not fully understood. To gradually increase the number of successful cases of medical devices developed in Japan, it is necessary to build a development model, expand it to other geographical areas, such as Shinshu, Oita, and Tottori, and support the development of CDs. Additionally, it is important to develop a knowledge base while utilizing the advantages of the local characteristics of these areas.
\end{abstract}

Keywords: industry-academia collaboration, medical-engineering cooperation, small and medium sized enterprise (SME), industry-academia collaboration coordinator (CD), medical device development support.

Adv Biomed Eng. 9: pp. 112-116, 2020.

Received on January 22, 2020; revised on March 30, 2020; accepted on April 20, 2020.

* Cooperative Major in Medical Photonics, Hamamatsu University School of Medicine, Hamamatsu, Japan.

\# 1-20-1 Handayama, Higashi-ku, Hamamatsu 431-3192, Japan. E-mail: amanoy@hama-med.ac.jp access article distributed under the terms of the Creative Commons BY 4.0 International (Attribution) License (https:// creativecommons.org/licenses/by/4.0/legalcode), which permits the unrestricted distribution, reproduction and use of the article provided the original source and authors are credited.

\section{Industry-Academia Collaboration in Hama- matsu}

Upon approval by the Cabinet in May 2016, the Japanese Government passed the "Basic plan for promotion of research and development and diffusion of medical devices to improve the quality of medical care received by the people" and enacted the necessary law in June [1]. It was determined that the medical device industry would be the driving force behind the domestic economy and that it could be an industrial sector that might achieve further growth and development in the future. The domestic market for the medical device industry is gradually growing every year. The shipment values of imported 
goods have increased, and the export values have remained flat, thereby creating excess imports. The imported proportion of high-class therapeutic devices and diagnostic devices is presently high, and the international competitiveness of Japanese companies is particularly weak in the field of therapeutic devices [2,3].

According to the evaluation data of the Networks for Supporting Development of Medical Devices, which was established after a cabinet decision, approximately $73 \%$ of the consulters were small and medium enterprises (SMEs) [2]. This is a network between Japan Agency for Medical Research and Development (AMED) and Mitsubishi Research Institute, Inc., which promotes collaborations between the national government and local regions such that medical device developers, including newcomers to the market, can receive extensive support for pharmaceutical affairs, intellectual property, technology, and marketing in an integrated manner. The consultations involve business strategies, technological developments from companies specializing in automotive parts and precision processing, regulatory compliance (classification including judgment as medical or non-medical device, and application procedures), market development (including sales agent information and development in overseas markets), and technological support (such as evaluations of technologies and prototypes). The motivation behind this work was to introduce collaborative examples from regions that have implemented advanced initiatives for medical device development, which would then provide guidance for efforts in other regions.

The development of medical devices using the Stanford Biodesign technique was introduced to Japan by the US and is a popular field in Japan in recent times [4]. In urban areas such as Tokyo and Osaka, foundations encouraging innovation were formed. These organizations have human resources and funding that can support the basic processes of identifying, inventing, and implementing new products, and aim to develop innovative medical devices. Kashino et al. [5] argue that such development will also require the trio of clinical needs, business know-how, and manufacturing technology [i.e., marketing authorization holder (MAH) driven model].

However, it is difficult to adopt these methods in local cities. For SMEs that are new entrants in the medical device field, even entry-level product offerings are unclear owing to differences in business structures. More than $30 \%$ of such companies are often unsure of their business goals [6]. There is also a need to determine the potential company needs. Therefore, universities, SMEs, and companies that lack market presence in the medical device field and are unfamiliar with medical device development are more likely to develop devices that are not innovative or do not serve specific purposes. Thus, there is a need to support these companies through an industry-academia collaboration coordinator (CD). In the policies and measures of the basic plan, it is important to match the advanced manufacturing technologies of SMEs with the needs of the medical practice and support SMEs that have not been previously involved in medical device development. It is therefore necessary to also find support organizations and specialists who are closely related to the field and available locally [1]. To verify this hypothesis, in the present study, a local city by the name of Hamamatsu that is engaged in medical-engineering collaborations was surveyed.

Figure 1 shows the Hamamatsu method. Industryacademia collaboration coordinators (CDs) assumed the lead in creating an ecosystem in which education, industry-academia and medical-engineering collaborations, funding, R\&D support, technology transfer support, and sales promotion support could be freely circulated [7]. From these observations, the case for acquiring competitive funding from joint research initiatives between universities and industry, by producing prototypes, by applying for medical devices, for commercializing projects, and for marketing products is introduced.

\section{Successful Examples of the Hamamatsu Method}

\subsection{Digital Laryngeal Strobe LS-H10}

Based on the medical needs of the Hamamatsu University School of Medicine (HUSM), NST Co., Ltd., developed a machine control device; Amelio Co., Ltd. developed the software; and Nagashima Medical Instruments Co., Ltd., commercialized the product [8]. The system developed is a laryngeal observation endoscope that uses sound and light control technologies to record and evaluate vocal cord movements. The inventors did not apply for a patent and handled it only as know-how. Instead, HUSM and Nagashima Medical Instruments Co., Ltd., signed a know-how license agreement. Manufacturing and sales certification of the system was obtained in March 2013, and marketing was commenced in 2015. As a low priced and popular compact product, it has since then been used in medical facilities.

The CDs translated the needs of the doctors and introduced them to local SMEs, creating a project team. In parallel, they aimed to create a joint team of the MAHs. They strategized technological achievements as knowhow rather than patents, and signed contracts between the universities and companies.

\subsection{Functional Inspection Oximeter KN-15}

Based on a joint research between HUSM and Shizuoka University, ASTEM Co., Ltd. commercialized a newly 


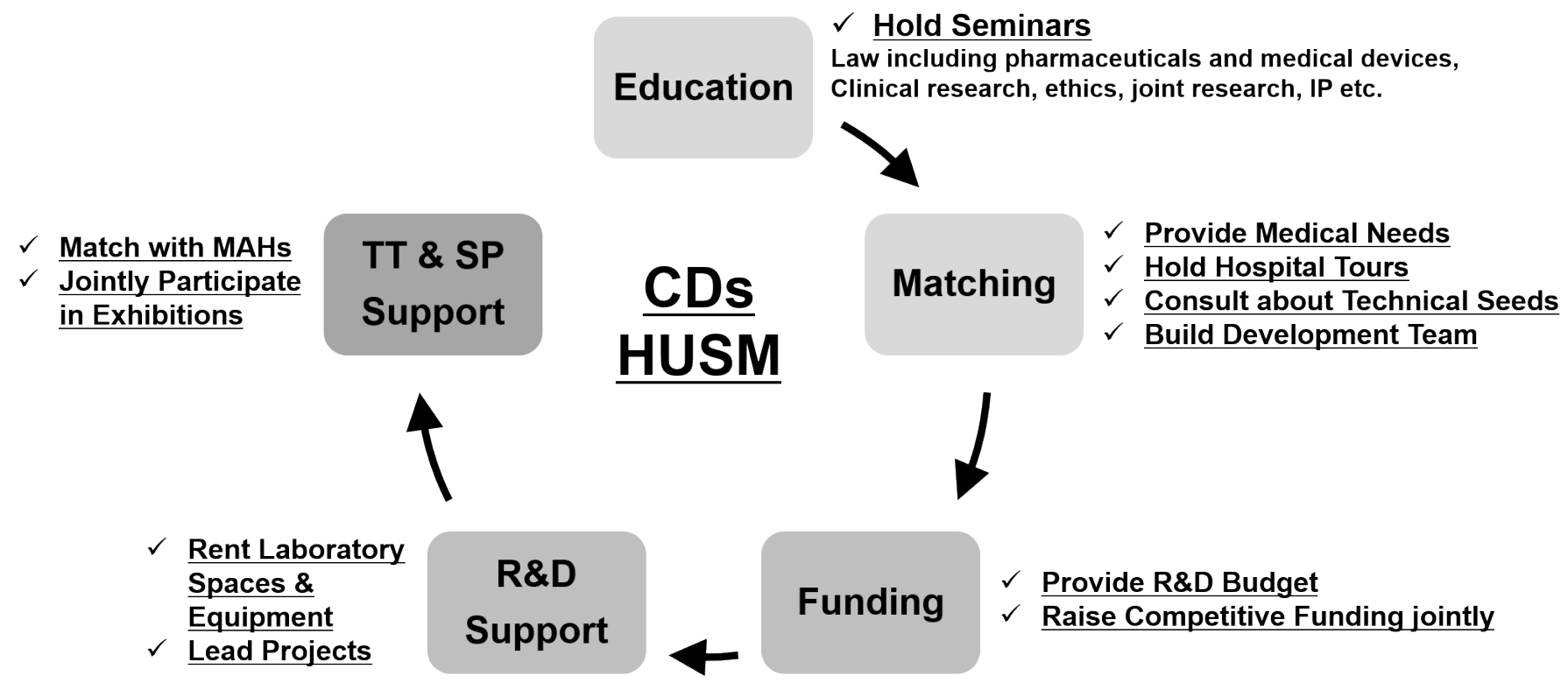

Fig. 1 Ecosystem of Medical Device Development.

CDs of Hamamatsu University School of Medicine (HUSM) provide the ecosystem to local SMEs.

TT: technology transfer; SP: sales promotion.

developed oximeter [9]. The device measures oxygen saturation $\left(\mathrm{rSO}_{2}, \%\right)$ and total hemoglobin index (T-Hbl) of the patient's blood by simply reading from a sensor probe placed on the finger. The two universities filed a patent application, and concluded a patent license agreement with ASTEM Co., Ltd. The three parties then applied for and acquired competitive funding to proceed with the R\&D efforts. ASTEM Co., Ltd. acquired the manufacturing and sales certification in January 2015 and commenced sales of the device. The initially intended use for this device was topical readings from newborns and adults, but were later changed to allow use in the oral cavity and vagina (birth canal) in 2019. The development team improved the device and filed new patent applications. They also obtained competitive funding and are preparing clinical trials for the new uses.

The CDs linked the needs of the doctors with the technological providers and supported patent applications through academic collaborations. They transferred technology to the company and assisted with signing contracts. They also helped to obtain competitive funding and attended consultations with Pharmaceutical and Medical Devices Agency (PMDA) and third-party certification bodies. Thus, the CDs were involved in building the R\&D strategy that included support for clinical trials.

\subsection{Bite Guard}

Based on the medical needs of HUSM, Shoda Techtron Corp. developed and manufactured a bite guard, and System JP Co., Ltd. handled the sales [10]. Currently, Medical Leaders Co., Ltd. is also a sales partner. In addi- tion to the function of the conventional bite block, the bite guard allows bite blocking with a function to protect the tongue and lips. HUSM and Shoda Techtron Corp. were both involved in acquiring competitive funding and device development. Both parties filed patent applications. System JP Co., Ltd. filed a manufacturing and sales application for this product in June 2016 and commenced sales shortly thereafter. The CDs translated the needs of the doctors and introduced them to local SMEs, thereby creating a project team. They also supported patent application.

\section{Discussion}

In the Hamamatsu area, R\&D efforts through industryacademia collaboration based on medical needs are continuously underway with the support of CDs. Examples of product development include colposcopes, hip prosthesis systems, and radiation therapy mouthpieces (certified). In the field of medical device peripherals and the field of care and welfare, product development includes marking systems for medical devices, work support system for medical workers, medical tray, rehabilitation device, and elastic stockings.

On the other hand, there were several cases of commercialization failure due to lack of effort. Broadly categorized, there were two patterns: when the needs of the MAHs could not be read, and when the medical and technical needs were not fully understood. In both cases, there were no or insufficient interventions from the CDs, and the collaborations were abandoned. 


\subsection{Cases Where the Needs of the MAHs Could Not Be Read}

The Hamamatsu area is a barren area for MAHs. In most cases, universities and SMEs are ignored even when they initiate talks with the MAHs in the stage of establishment of the medical and technical needs. Therefore, the developers searched for manufacturers after filing patent applications and creating prototypes. They sought MAHs and attempted to establish joint development efforts. The creators had several interviews, but the MAHs declined because the creations were not in line with their development strategies, and only information extraction was achieved. In addition, because the MAHs took a long time for internal review and did not contact the creators, the development period was extended and the SMEs withdrew. This was especially the case when trying to develop high-class medical devices.

\subsection{Cases Where the Medical and Technical Needs Were Not Fully Understood}

The SMEs heard about certain medical needs from doctors and medical workers and assumed that these were absolute or critical requirements. On the basis of their assumptions, they applied for patents and proceeded with development. Even when they participated in exhibitions before sales or immediately after commencing sales, the reactions of the visitors were weak. At this stage, they conducted a market survey and found a gap between their product and medical needs, and they stopped the development and sales. In some cases, they were delayed in the development stage and could not keep pace with the changing medical needs. There were also cases where doctors were unable to fully understand the technical seeds and companies withdrew owing to poor communication.

\subsection{Observations and Conclusions}

For medical device development in the Hamamatsu area, the success rate from technical consultation with SMEs to completion of prototype was $19 \%$, and that to commercialization was $5 \%$. The average period from technical consultation to commercialization was 3 years. In both cases, the universities and SMEs jointly acquired competitive funds for R\&D. Although general comparisons cannot be made, the development of a medical device costs on average several million yen to several billion yen and takes 6 years to commercialization. In the Hamamatsu area, efficient development has been achieved in a shorter period of time. The CDs provided hands-on support in all cases in which they assisted with commercialization efforts. The success factor in the Hamamatsu area is presumed to be because the ecosystem (Fig. 1) fostered by the CDs was utilized during de- velopment. The key to the success of the digital laryngeal strobe was that the CDs avoided patent application that was a hurdle for the SMEs. Instead, they proposed and implemented a mechanism that would increase the profits of the SMEs from selling these products. In the case of the functional inspection oximeter, the CDs balanced the power relationship between the medical and engineering departments, while the university/hospital and SMEs maintained a mutual trust relationship even after the product was commercialized and established steady sales. In the case of the bite guard, the CDs supported the goal to increase sales as well.

On the other hand, in the failure cases, doctors or medical workers and SMEs could not take over the development from the MAHs (when the needs of MAHs could not be read). As a solution, the MAH-driven model can be considered, in which MAHs are added to the team at the initial stages of R\&D [5]. In Tokyo, there is a cluster study group that performs direct matching between doctors or medical workers and MAHs, and searches for SMEs that supply parts after the matching. The cluster study group requires that patent applications be filed before announcing the medical needs [11]. MAHs sometimes refused joint research because they assume that $\mathrm{R} \& \mathrm{D}$ cannot be controlled if patents are filed in advance. Hence, the MAH-driven model is not suitable because doctors or medical workers and SMEs have common goals in the Hamamatsu area.

One of the other causes of failure is that the SMEs did not respond to changes in medical needs and diversity (when the medical needs and technical seeds were not fully understood). As a potential request, SMEs vaguely desired to develop medical devices [6]. On the other hand, doctors and medical workers were unable to quantify their true needs. For this reason, SMEs misunderstood the unsophisticated personal needs as absolute medical needs. There were many other cases where medical needs were clarified and product concepts refined through prototype production and repeated discussions between doctors or medical workers and SMEs. The greatest deterrent to medical device development through industry-academia collaborations is the lack of communication. However, too much input can impede development and progress. It is also the role of the CDs to coordinate mutual communication. A doctor's ability to communicate is also a critical factor; doctors who were famous in the past do not always continue to be experts in their field and do not always understand current medical needs. An important role of the CDs is to create an environment in which all concerned parties can contribute equally.

In order to continuously increase the success rate of medical device development in Japan, it is necessary to 
build a development model that can be sustainably deployed in other geographical areas, while fostering CDs. In other cities such as Shinshu, Oita, and Tottori, efforts are being made in a similar manner as in the Hamamatsu area. These require additional know-how while improving cooperation between areas and utilizing the unique local characteristics of each area.

\section{References}

1. Cabinet decision: Basic plan for promotion of research and development and diffusion of medical devices improve the quality of medical care received by the people: <https://www.mhlw.go. jp/file/04-Houdouhappyou-10807000-Iseikyoku-Keizaika/ 0000125967.pdf $>$ [accessed on January 12, 2020].

2. The Japan Federation of Medical Device Association: Overview of domestic production dynamics in the medical device industry: $<$ http://www.jfmda.gr.jp/device/industry/> [accessed on October 12, 2019].

3. Ministry of Economy, Trade and Industry: About the medical device industry policy in METI: <https://www.med-device.jp/ repository/meti-seisaku-201909.pdf $>$ [accessed on October 27, 2019].

4. York P, Makower J, Zenios S, Biodesign: The Process of Innovation Medical Technologies. Cambridge University Press. 2015.

5. Kashino T: Intellectual property strategy for medical device development: 16th Annual Meeting of Japan Society for Intellectual Production. 2018.

6. Headquarters for Healthcare Policy, Cabinet Office, Ministry of Education, Culture, Sports, Science and Technology, Ministry of Health, Labour and Welfare, and Ministry of Economy, Trade and Industry: Medical device development support handbook: $<$ https://www.med-device.jp/repository/handbook20190326. pdf $>$ [accessed on October 27, 2019].

7. Hamamatsu Medical \& Engineering Technology Innovation Core: Annual Report in 2014-2018: 2015-2019.

8. Nagashima Medical Instruments Co., Ltd.: Digital Laryngeal Strobe LS-H10: <http://www.nagashima-medical.co.jp/ content/view/692/66/> [accessed on October 12, 2019].

9. Astem Co., Ltd.: Toccare KN-15: <https://astem-jp.com/ product/toccare/> [accessed on October 12, 2019].

10. System JP Co., Ltd.: Bite Guard: <http://www.sysjp.com/ biteguard.html $>$ [accessed on October 12, 2019].

11. Kanto Bureau of Economy, Trade, and Industry: Intellectual property of medical and engineering cooperation learned from cases: <https://www.kanto.meti.go.jp/seisaku/iryokiki/data/ 20190410_chizai_katsuyou_sasshi.pdf> [accessed October 27, 2019].

\section{Yuko Amano-Ito}

Yuko AмANo-Iтo received master's degree from the University of Tokyo in 2004. She is currently a student at doctoral program in Cooperative Major in Medical Photonics, Hamamatsu University School of Medicine. Her research interests include intellectual property and industry-academia collabora-

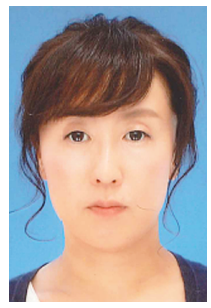
tion. She is a member of Japan Society for Medical and Biological Engineering. 